\title{
Linx
}

Revue des linguistes de l'université Paris X Nanterre

$11 \mid 1999$

Typologie des langues, universaux linguistiques

\section{Le marquage des subordonnées « de circonstance »: marques segmentales et non segmentales en malgache}

\section{Huguette Fugier}

\section{OpenEdition}

\section{Journals}

Édition électronique

URL : http://journals.openedition.org/linx/891

DOI : 10.4000/linx.891

ISSN : 2118-9692

Éditeur

Presses universitaires de Paris Nanterre

\section{Édition imprimée}

Date de publication : 1 décembre 1999

Pagination : 125-138

ISSN : 0246-8743

\section{Référence électronique}

Huguette Fugier, «Le marquage des subordonnées « de circonstance » : marques segmentales et non segmentales en malgache », Linx [En ligne], 11 | 1999, mis en ligne le 29 juin 2012, consulté le 20 avril 2019. URL : http://journals.openedition.org/linx/891; DOI : 10.4000/linx.891

Ce document a été généré automatiquement le 20 avril 2019

Département de Sciences du langage, Université Paris Ouest 


\title{
Le marquage des subordonnées « de circonstance »: marques segmentales et non segmentales en malgach $e^{1}$
}

\author{
Huguette Fugier
}

\section{NOTE DE L'ÉDITEUR}

Les textes cités sans référence sont des expressions courantes ou ont été produits par des malgachophones au cours d'entretiens.

1 La syntaxe malgache comporte différentes sortes de $\mathrm{P}$ dépendantes: complétives introduites par hoe, $f a, h o$, mais aussi subordonnées servant à exprimer les circonstances de "temps", "cause ", " condition »... Parmi ces dernières, rares sont celles qui se laissent comparer aux « circonstancielles » des grammaires européennes, c'est-à-dire qui à la façon de la $\mathrm{P}(1)$ ci-dessous juxtaposent simplement, à une conjonction de " circonstance ", une P par ailleurs ordinaire. L'opérateur de subordination ne consiste généralement pas en un quelconque morphème segmental qui vienne s'adjoindre de l'extérieur à l'ensemble des constituants formant la $\mathrm{P} 2$ : et là où existe un tel relateur, il convient de préciser quel rôle exact joue celui-ci dans l'opération qui fait la subordonnée «de circonstance ».

2 Mais avant d'interpréter on s'imposera, comme il se doit, de bien décrire les faits. Un premier paragraphe énumérera donc tous les schémas de P2 «circonstancielles » que livre un corpus apparemment représentatif de cet état de langue dit classique moderne ou malgache officiel. Des schémas structuraux ainsi offerts à la comparaison, un paragraphe 2 essaiera de tirer quelques enseignements sur les mécanismes de la subordination en malgache. 


\section{Les réalisations effectives : inventaire}

Après avoir exemplifié par la $\mathrm{P}(1)$ un type de subordonnée conjonctive interprétable d'emblée par tout locuteur d'une langue européenne, mais qui ne réalise pas la structure la plus originale du malgache, nous présenterons l'ensemble des autres types en deux groupes, respectivement :

- - introduit par un relateur : $\mathrm{P}(2)$ à (5)

- - dépourvu de tout relateur introductif : P(6) et (7).

4 ANALYSE. La P2 introduite par le relateur satria « parce que » se présente ici dans l'ordre non-marqué

manavy + zana-ny

préd. + suj.

5 Mais l'ordre inverse (suj.+ préd.), considéré comme marqué, n'est pas moins licite ; auquel cas, le suj. initial se voit souvent suivi de no -ce connecteur qui sert à désigner le suj. comme terme distingué de la $\mathrm{P}$, et que l'on verra significativement exclu de toutes les structures (2) à (7) :

\section{(1.a) Tsy afaka Rakoto satria ny zana-ny no manavy.}

En résumé,

SCHEMA DE LA P(1) :

relateur

et une P2,

-avec ordre libre pour les 2 constituants suj. et préd.

-connecteur no admis (après suj. initial, c.à.d. dans l'ordre : suj.

+ préd.).

(2.1) Miarahaba alny izy isaky my mifanena izahay saluer moi il chaque fois (article) se rencontrer nous $=$ " Il me salue chaque fois que nous nous rencontrons" (RAJEMISARAOLISON : 152).

(2.2) Ary tonga nitondra tafika ... raha ilay et venir amener armée quand (démonstratif)

niakatra tany Mitongoa iny Rahasamanarivo
monter là

$=$ « Rahasamanarivo vint en amenant une armée quand il monta à Mitongoa » (RAMAROSON-GIAMBRONE : 28).

ANALYSE. En (2.1), le relateur précède une séquence de 3 termes : ny, mifanena, izahay. De ceux-ci, les 2 derniers constituent évidemment le préd. et le suj. de la P2, avec cet ordre constant sans possibilité de l'ordre inverse (suj. + préd.). Quant à ny, cet article tient sa raison d'être de la syntaxe plutôt que de la sémantique, c'est-à-dire que loin de « définir » 
ou « déterminer » quoi que ce soit il sert à saisir la P2 subséquente pour rendre possible son insertion dans la position c.c. de la P1 incluante.

7 En (2.2), à la suite du relateur raha c'est la paire de démonstratifs ilay ... iny qui encadre le préd.2 niakatra -laissant à l'extérieur le suj. commun à P2 et P1, Rahasamanarivo.

En résumé,

SCHEMA DES P(2):

relateur

et

$$
\begin{aligned}
& \text { en (2.1) article + (préd. + suj.) } \\
& \text { en (2.2) (démonstratif + préd. + démonstratif })+ \text { suj. }
\end{aligned}
$$

(3) Ny aretim-po-ny nihetsika tampoka tamin' izy torana

la maladie-coeur-de-elle bouger soudain lors de elle évanouie

$=" \mathrm{Sa}$ maladie de coeur évolua soudain à l'occasion de son évanouissement " (TREZAN in RAJAONA, $\mathrm{n}^{\circ}$ 11: 101).

$\begin{array}{llllll}\text { - Efa nafoi-ko } & \text { avokoa } & \text { ny trosa-kao } & \text { rehetra } \\ \text { déjà } & \text { aboli-par-moi tout } & \text { le dette-de-toi tout }\end{array}$

$\begin{array}{lllll}\text { tami-nao } & \text { noho } & \text { ianao } & \text { nifona } & \text { tami }-k o \text {. } \\ \text { à }- \text { toi } & \text { à cause de } & \text { tu } & \text { supplier } & \text { à }\end{array}$

$=\ll$ Je viens de te remettre toutes tes dettes à la suite de tes supplications

auprès de moi " (ex. de RAHAJARIZAFY s.d. : § 1002).

8 ANALYSE. Dans ces deux réalisations $\mathrm{P}(3)$ structurellement identiques, le relateur tamina /noho précède une séquence constituée chaque fois

\begin{tabular}{|l|l|l|}
\hline d'un N/pronom & et & d'un Adj./V \\
\hline izy & & torana \\
\hline ianao & & nifona. \\
\hline
\end{tabular}

Si l'on admet que ces deux constituants forment ensemble une $\mathrm{P}$ (suj. + préd.) globalement saisie par l'opérateur de subordination tamina/noho, alors les $\mathrm{P}(3)$ ne s'écartent pas du schéma 2 ci-dessus. Pourtant, l'ordre des mots fait objection à cette interprétation spontanée. Car l'ordre constant suj. + préd. (izy torana, à l'exclusion de torana izy ), du seul fait qu'il s'oppose à l'ordre inverse préd. + suj. obligatoire dans les $\mathrm{P}$ (2), signale assurément une différence entre les deux structures. Préférant donc une autre solution, nous considérerons que le relateur saisit seulement le N/pronom -comme noho fait par exemple de ianao en :

$$
\begin{aligned}
& \text { (3.a) Noho ianao, avela - ko } \quad \text { ny helo - ny } \\
& \text { à cause de toi } \quad \text { pardonné-par-moi le faute-de-lui } \\
& \text { = «A cause de toi, je pardonne sa faute } \text { (ex. de RAHAJARIZAFY 1960: 64) ; }
\end{aligned}
$$

lequel N/pronom s'adjoint éventuellement une expansion épithétique. Que cette dernière se réalise parfois par un $\mathrm{V}$ (nifona ) n'étonnera pas, dans une langue où le $\mathrm{V}$ s'appose régulièrement à un $\mathrm{N}$-support, comme l'exemplifie mandeha épithète de olona en : 


\section{(3.b) Ny olona mandeha an-tongotra dia maro-be le personne aller à-pied nombreux $=$ "Les gens allant à pied sont nombreux $"$.}

10 La construction tamin' izy torana = litt. «à l'occasion de elle évanouie », noho ianao nifona ami-ko = litt. «à cause de toi m'ayant supplié», rappellera finalement au lecteur francophone cette tournure de langue parlée :

\begin{tabular}{|l|l|l|l|}
\hline & relateur + & $(\mathrm{N}$ & + épithète $)$ \\
\hline (Je suis en retard) & à cause de & mon petit & qui est malade \\
\hline \hline (Je suis tombé) & à cause de & lui & qui m'a poussé \\
\hline (C'est arrivé) & au moment de l'orage & qui a éclaté. & \\
\hline
\end{tabular}

11 La place linéaire de torana/nifona derrière izy/ianao est alors tout normalement celle de l'épithète après son $\mathrm{N}$-support.

12 Remarque. La forme aho/ianao/izy employée dans les $\mathrm{P}(2)$ de préférence à ahy/anao/azy n'en fait pas nécessairement des sujets d'un prédicat. La série aho..., plutôt qu'elle ne code la fonction syntaxique sujet, réalise de façon plus générale des formes non gouvernées (comme j'ai essayé de le montrer ailleurs : Syntaxe malgache, Louvain, Peeters, 1999 : 22). De toutes façons, le choix de aho/ianao/izy s'impose dans le dispositif des P(3) par l'effet de la même contrainte qu'on voit jouer par exemple en :

\section{(3.c) Ny tranon' izy mivady \\ le maison-de- eux époux \\ $=$ litt. « La maison de eux-époux » c.à.d. "La maison des époux ",}

13 en vertu de ce fait qu'un pronom lui-même en état de dépendance garde sa forme non gouvernée s'il se trouve suivi d'une expansion.

14 En résumé, 
SCHEMA DE LA P(3) :

relateur

et $\mathrm{N} /$ pronom dans sa forme non-gouvernée + son expansion épithétique (adjectivale ou verbale).

(4.1) Raha tsy ianao, tsy namela heloka aho si ne..pas tu ne...pas pardonner faute je $=$ "Si ce n'était toi (s'il n'y avait toi), je ne pardonnerais pas la faute".

- Raha izaho

si je

$=\ll$ S'il s'agit de moi, quant à moi $»$.

- Satria ianao

parce que tu

$=$ « Parce que c'est toi, parce qu'il y a toi $»$.

(4.2) Ambonin' izany dia nymarakely fanaon' nympianatra

en plus de ceci fait-par-le élève

isaky ny fetin' ny Repoblika frantsay

chaque fois fête République français

$=$ = En plus, le marakely exécuté par les élèves chaque fois que (c'était)

fête de la République française" (RATSIFANDRIHAMANANA,

Hiakatra ny Anglisy ! : 76).

15 Note. Marakely: sorte de spectacle, où les jeunes garçons exécutent des mouvements rythmiques en brandissant des bâtons.

ANALYSE. Introduit par un relateur et revêtant sa forme non gouvernée ianao /izaho, le pronom des $\mathrm{P}(4.1)$ constitue à lui seul la $\mathrm{P} 2$ entière : situation normale en malgache, où le $\mathrm{N}$ isolé suffit à faire Phrase (cf. Tsena = litt. "Marché » c.à.d. « Il y a marché, c'est le marché... »). Comment prendre d'ailleurs, dans l'énoncé donné, ce ianao/izaho pour le suj. d'un préd. inexistant, ou le préd. d'un suj. inexistant ? De toutes façons le ianao préd. d'un éventuel suj. recevrait l'interprétation identifiante " $N$ est (la même personne que) toi », exemplifiée par (4.a), fort différente de celle attachée en fait à (4.2) :

$$
\begin{aligned}
& \text { (4.a) Raha tsy ianao io sary io } \\
& \text { si ne..pas tu ce photo ce } \\
& =\text { "Si cette photo n'est pas toi..." }
\end{aligned}
$$

17 C'est encore une P2 complète que réalise fety en (4.2) -un N prototypique cette fois. L'article ny opère, ici comme en (2), sur le Nom-Phrase, pour l'habiliter à venir occuper sous P1 une position de circonstant; et s'il manque en (4.1) c'est seulement par l'incapacité générale des pronoms personnels (par eux-mêmes auto-définis) à accepter quelque article que ce soit. En résumé, 


(5.1) Vao mainka nitombo moa ny fandrosoana taorian'
encore plus
s'accroître (assertif) le progrès $\begin{aligned} & \text { après } \\ & \begin{array}{l}\text { ny namoahana } \\ \text { publication }\end{array} \text { ce lalàna } \\ & \text { cei }\end{aligned}$

= "Les progrès s'accentuèrent encore après qu'on eut publié cette loi " (RAMAROSON-GIAMBRONE : 77).

- Mialà tsiny ami-ko hianao noho ny tsy nampandrenesa enlève! blâme de - moi tu à cause de le ne..pas faire-savoir

- nao ahy izao zava-nidona teto izao

- de-toi à moi ce chose- frappant ici ce

$=$ "Excuse-toi auprès de moi de ne mavoir pas fait savoir cette chose sensationnelle (survenue) ici » (RAPATSALAHY : 48).

(5.2) Hatramin' izay nahasitrahan-dRabe iny izay dia izy depuis ce guérir - de - voici ce il

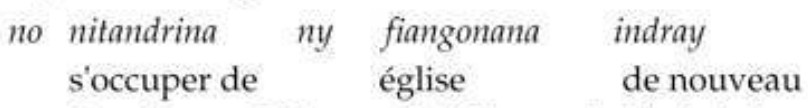
$=$ "Depuis que Rabe a guéri, il a repris l'administration de la paroisse " (exemple de RAHAJARIZAFY s.d. : §1166).

ANALYSE. En (5.1) le N verbal namoahana /nampandrenesana, litt. « le fait de publier/ de faire savoir " constitue à lui seul la P2. L'article qui l'introduit produit le même effet qu'en (2) et (4.2) ci-dessus. Il en va de même en (5.2), avec cette seule différence que le N verbal nahasitranana se trouve cette fois encadré entre les deux occurrences du démonstratif izay ... iny izay . En résumé,

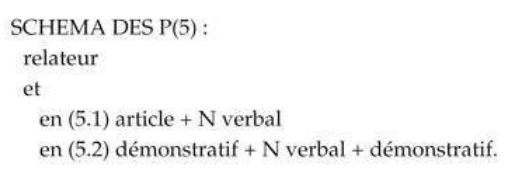

(6.1) Ity isika akaiky tobi-miaramila loatra ity notena ratsy ce nous près de camp-soldat trop ce précisément mauvais $=$ litt. $" \mathrm{Ce}$ (nous sommes trop près d'un camp militaire) est précisément mauvais " c.à.d. "Que nous soyons trop près d'un camp militaire, c'est bien ce qui est fâcheux" (RATSIFANDRIHAMANANA, Hiakatra ny Anglisy!: 83).

(6.2) Azo sambobelona nympanjaka, mbamin' ny vahoaka pris capturé vivant le roi avec peuple rehetra, afa-tsy zatovo maromaro izay tsy nahazaka entier sauf jeune homme nombreux qui ne..pas supporter izany ho entin-ko babo izany ce (futur) emmené-pour prisonnier ce

= litt. « Le roi fut capturé vivant avec le peuple entier, à l'exception d'un certain nombre de jeunes gens, qui ne pouvaient supporter ce (devoir être emmenés comme captifs) " (et se jetèrent du haut de la falaise) c.à.d. "qui ne pouvaient supporter la perspective d'être emmenés en captivité) " (RAMAROSON-GIAMBRONE : 37).

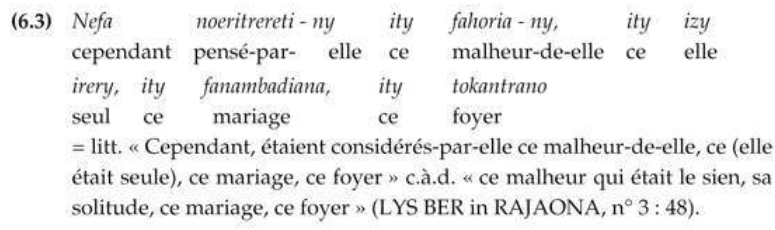


19 ANALYSE. En rupture avec les précédentes, les $\mathrm{P}(6)$ et (7) présentent des $\mathrm{P} 2$ dépourvues de relateur introductif. Elles ont en commun (outre l'usage déjà connu du démonstratif) l'ordre fixe des constituants en P2, soit: suj. + préd. Pour autant, les P2 respectives assument-elles forcément la même fonction dans la P1 incluante? Deux descriptions séparées sont nécessaires pour répondre à cette question.

En (6.1), l'ensemble (isika + akaiky) se trouve encadré par ity ... ity . En (6.2), izany ... izany encercle le préd.2 ho entin-ko babo -dont le suj. reste en ellipse, restituable à partir du zatovo inclus dans le SP afa-tsy zatovo maromaro . En (6.3), la P2 (izy + irery) ne reçoit qu'une occurrence du démonstratif : le ity antéposé ; elle aligne ainsi sa présentation sur les deux $\mathrm{N}$ ity fahoria-ny, ity fanambadiana qui la flanquent de part et d'autre et partagent sa fonction syntaxique en P1.

En cet équipage, la P2 des P(6) vient occuper une position d'actant dans le noyau de P1. En (6.1), (isika akaiky) sert de suj. au préd. ratsy, et en ordre linéaire il occupe cette place initiale à quoi succède le connecteur no marqueur typique du suj. emphatisé. En (6.2), ( entin-ko babo ) fonctionne en P1 comme c.d. du préd.1 nahazaka, et suit son V gouverneur comme ferait n'importe quel c.d. nominal. En (6.3), (izy irery ) s'insère dans une chaîne de Noms prototypiques, dont il partage la fonction de suj. en P1, et la place non-marquée, après le V1 passivé noeritrereti-ny . En résumé,

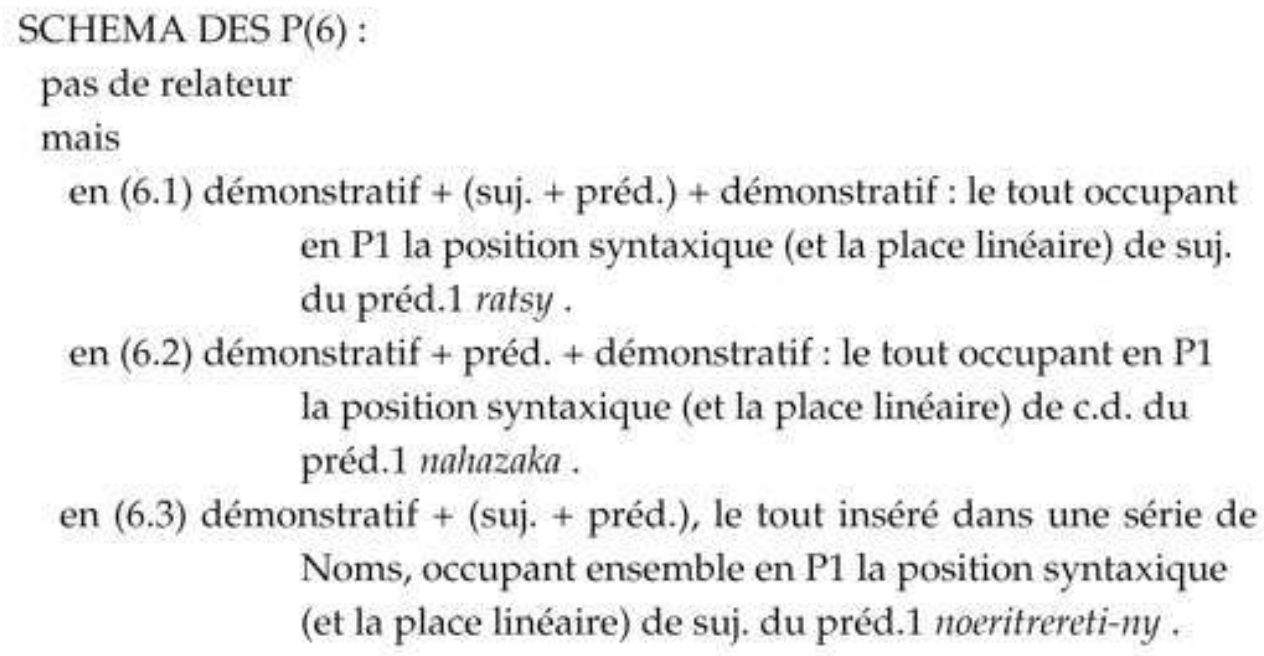

Les P2 rattachées au schéma (6) ne sont donc pas des subordonnées " de circonstance ». L'encadrement par démonstratifs ainsi que l'ordre singulier (suj. + préd.) les signalent, sans plus, comme autant de $\mathrm{P}$ dépendantes au sein d'une P1 incluante. Mais n'en va-t-il pas exactement de même pour les P2 relevant du type (7)? Quelles particularités s'ajoutent à ces dernières, qui fasse des $\mathrm{P}(7)$ proprement des "circonstancielles»? Voyons les énoncés :

(7.2) diffère de (7.1) par ceci que le démonstratif y encadre le seul préd.2 nitodika, laissant extraposé à droite du second iny le suj. ravehivavy commun à P2 et P1 -suj. toutefois inclus dans P2 comme le montre la place de la pause et de l'éventuel no (mentionné ci-dessous). Pour tout le reste les deux P partagent les mêmes caractéristiques : 

(7.1) Izao isika miteny izao dia efa loa-jafy ryMose ce nous parler ce déjà grand-parent Monsieur

\section{sy Madama Rabeloha}
et Madame
$=" \mathrm{Au}$ moment où nous parlons, Monsieur et Madame Rabeloha sont déjà grands-parents " (RAILOVY in RAJAONA, $n^{\circ}$ 19: 214).

$\begin{array}{llllll}\text { (7.2) Iny nitodika } & \text { iny } & \text { ravehivavy, maty } & \text { tery } \\ \text { ce } & \text { se retourner } & \text { ce } & \text { femme } & \text { mort } & \text { aussitôt }\end{array}$

= «A l'instant où la femme se retourna, elle mourut aussitôt " (exemple de RAHAJARIZAFY s.d. : §1137).

ANALYSE.

24 - Par rapport à P1, les P2 présentées en (7.1) et (7.2) se situent en position externe hors du noyau, et plus précisément ici en tête de Phrase sans possibilité d'aucun déplacement linéaire. L'absence de relateur entraîne pour conséquence que faute de conjonction capable de fournir un sens lexématique précis tel qu' "antériorité/postériorité", «condition »..., l'interprétation «procès simultané à celui de P1» s'impose de toutes façons : «Au moment où nous parlons... », «A l'instant où la femme se retourna... ».

- Avec sa P1 subséquente, la P2 n'a pas d'autre lien formel que la simple juxtaposition. Certes les démonstratifs encadrants et l'ordre fixe (suj. + préd.), comme on a vu plus haut, la signalent et la maintiennent en l'état de $\mathrm{P}$ dépendante. Il y a pourtant des degrés dans cette dépendance, et sur ces variations les connecteurs apportent un utile témoignage. .. (7.1) comporte le connecteur dia, et dans le schéma ainsi réalisé (P2 dia

P1), n'importe quel SP (préposition + N) commuterait avec P2.

.. (7.2) admet le connecteur no :

\begin{tabular}{|l|l|} 
(7.a) & Iny nitodika iny ravehivavy no maty tery \\
\hline & = « La femme ne se fut pas plutôt retournée qu'elle mourut », \\
\hline
\end{tabular}

et rappelle en ceci les paires de P corrélées, telles que peut lesexemplifier

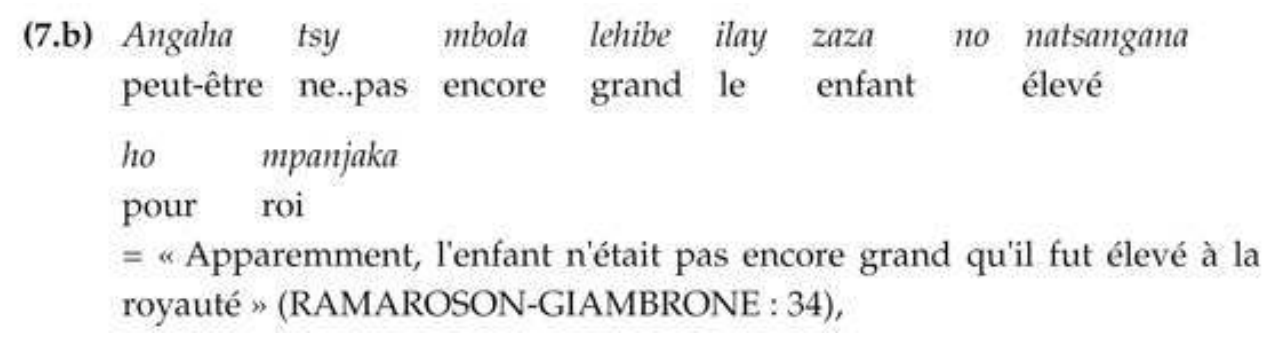

sans que pour autant notre (7.2), avec sa P2 marquée comme dépendante et incapable de subsister seule, réalise vraiment ce schéma structural de deux $\mathrm{P}$ équifonctionnelles liées par no (et par un contour intonationnel unifiant) qu'on peut nommer schéma de corrélation. La juxtaposition sans relateur d'une $\mathrm{P}$ dépendante auprès de sa principale autorise, sans plus, de l'une à l'autre une relation plus ou moins serrée.

En résumé, 


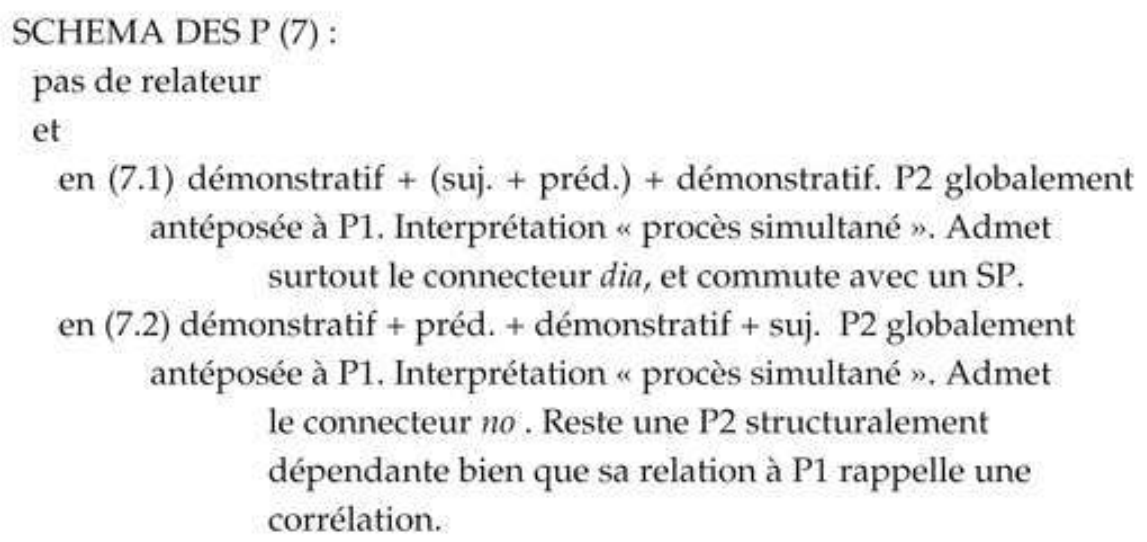

\section{Interprétation} autre que l'article/démonstratif, qui intervient pour saisir un ensemble de constituants formant Phrase, et lui permet par ce moyen de prétendre à une des positions syntaxiques qu'offre le schéma de la $\mathrm{P}$ super-ordonnée. On dira que cet article/démonstratif 
recatégorise l'unité syntaxique ainsi saisie en ce sens que la prenant en l'état de Phrase échappant encore à toute subordination il la rend capable d'accéder au statut de constituant dans une autre $\mathrm{P}$ incluante. Un tel opérateur intervient, dans les types de $\mathrm{P}$ considérés aujourd'hui, de façon nécessaire puisqu'aucune des P2 décrites ne serait en état $\mathrm{d}$ ' atteindre sans lui à des positions en $\mathrm{P} 1$-tant positions d'actants comme en (6) que de circonstants comme en (7) : et de façon suffisante puisqu'efficace en l'absence de tout relateur, comme le montre la série (6)-(7).

A cet effet opératoire qu'il partage avec l'article, le démonstratif joint la prestation de quelques services qui lui sont propres. De fait :

- Il ajoute du sens. Ainsi izany de (6.2) n'équivaut-il pas exactement à ity de (6.1), ainsi iny de

(5.2) apporte-t-il la connotation « (cette guérison) déjà mentionnée, dont vous avez connaissance ».

- Il sert de démarcation, quand sa deuxième occurrence limite sur la droite la P2 pour faciliter au décodeur la segmentation de l'énoncé complexe (P2 dans P1) -un service dont le § 2.2.2. ci-dessous montrera l'utilité.

Mais si l'article/démonstratif suffit à opérer la subordination, à quoi servent donc les relateurs ? Quelle utilité reste à isaky/raha/tamina ... qui introduisent les P2 en (2)-(5) ? Il leur reste la fonction propre de gouverner la P2. Gouverner n'est pas subordonner. Le relateur prend en charge la $\mathrm{P} 2$ déjà saisie par l'article/démonstratif, c'est-à-dire rendue capable de s'insérer dans une position syntaxique, et l'insère donc effectivement en P1 dans une position de circonstant. En quoi la dénommée «conjonction» n'agit pas autrement que la "préposition» devant un $\mathrm{SN}$ (article/démonstratif $+\mathrm{N}$ ), et aucune différence structurale ne sépare entre eux les membres de la série

\begin{tabular}{|l|l|}
\hline avec N prototypique & taorian' ny ady = « après la guerre » \\
\hline \hline avec N verbal & taorian' ny namoahana en (5.1) \\
\hline avec Nom-Phrase & isaky ny fety en (4.2) \\
\hline avec (N + son épithète) & tamin' izy torana en (3) \\
\hline avec P (préd. + suj.) & isaky ny mifanena izahay en (2.1). \\
\hline
\end{tabular}

40 Le relateur fournit en outre du sens. La valeur attachée au lexème, comme aoriana "après", isaky "chaque fois", raha "quand, si », satria "parce que», induit l'interprétation temporelle/conditionnelle/causale de la P2, tandis qu'à l'inverse l'absence de relateur n'autorise que l'interprétation suggérée par la simple co-présence de $\mathrm{P} 2$ à $\mathrm{P} 1$, soit : « procès $\mathrm{P} 2$ simultané au procès $\mathrm{P} 1$ ».

\subsection{2.}

41 Ainsi subordonnée et gouvernée, la P2 se voit signalée par des marques -positives ou négatives, utiles en tous cas au décodeur pour identifier sans erreur, soit la P2 par différence avec la P1, soit tel type de P2 par différence avec tel autre. Plus frappantes à première vue, les marques positives consistent en indicateurs démarcatifs et/ou distinctifs. 
42 - le rôle démarcatif revient aux démonstratifs dont la deuxième occurrence clôt la P2 sur la droite. La barrière ainsi posée indique le point où finit la $\mathrm{P} 2$, et ce bornage s'avère utile .. aux subordonnées occupant en P1 les fonctions actancielles de suj. ou c.d. car cellesci,incluses en P1 dont elles forment un constituant parmi d'autres, manqueraient sans cela de visibilité. On percevra par exemple en (6.1) la difficulté de segmenter la P2 (Ity isika akaiky ... loatra) vs la P1(tena ratsy) en l'absence du second ity (et du connecteur no). .. Mais aussi aux P2 «de circonstance» comme (2.2) ou (5.2); surtout lorsque ces dernières, dépourvues de relateur comme en (7), exigent au moins d'être bien bornées sur leur droite.

- Le rôle distinctif revient principalement à l'ordre des mots, en tant que :

. L'ordre fixe (suj. + préd.) singularise la P2 à l'encontre de l'ordre non-marqué (préd. + suj.) usuel en P1 : ceci, dans les types dépourvus de relateur comme (6) et (7) qui trouvent là leur principale marque de subordination.

47 .. L'ordre fixe (préd. + suj.) distingue le type de P2 illustré par (2) de celui qu'exemplifient les énoncés (3). En effet l'ordre (suj. + préd.), s'il se réalisait par exemple en (2.2) avec le résultat: ${ }^{*}$ Raha ilay Rahasamanarivo niakatra..., exposerait la séquence ainsi produite à recevoir la même analyse que (3), c'est-à-dire :

au lieu de l'analyse souhaitée : relateur + une P (constituée d'un suj. et d'un préd.)

l'analyse, certes justifiée pour (3) mais indésirable en (2) : relateur + un (pro)nomphrase (escorté de son épithète).

Parmi les marques négatives, la plus évidente est l'impossibilité d'introduire no après le suj. des P2 «de circonstance» . Le connecteur no postposé au suj., et désignant celui-ci comme terme distingué de la $\mathrm{P}$, trouve sa place normale dans les indépendantes et quelques types précis de subordonnées (complétives; conjonctives telles que la $\mathrm{P}(1)$ exclue de l'actuel exposé) : à bon droit puisque le suj. réalise en pareil cas le terme opérande auquel s'applique le préd.; et de ce fait, logiquement parlant, le support de l'assertion. Or, de nos P2 (2) à (7), celles mêmes qui comportent un suj. prédiqué (ce qui exclut déjà les types (3), (4) et (5)) ne remplissent pas les conditions qui feraient de leur suj. un terme emphatisé, ou feraient d'une telle P2 insérée en position dépendante le signifiant d'une assertion propre. L'absence, effectivement vérifiée, de no en ces P2, particularité négative qui vient en conséquence de leur statut subordonné « circonstanciel », les signale en tant que telles aux yeux du décodeur.

\subsection{3.}

Les procédés mis en oeuvre pour réaliser la subordination, les outils formels qui servent à cet effet, se font remarquer par leur mode d'intervention.

Les procédés interviennent en succession ordonnée. Ainsi les relateurs viennent-ils gouverner une $\mathrm{P} 2$ déjà saisie par l'article/démonstratif, et de ce fait déjà proprement subordonnée.

51 Les outils formels utilisés tout au long du processus subordonnant ne sont pas voués, chacun singulièrement, à une tâche unique. Le même peut au contraire rendre plusieurs services successifs, ainsi le démonstratif sert-il (commutant alors avec l'article) à recatégoriser une unité phrastique en possible constituant d'une $\mathrm{P}$ incluante, et (réitéré en deuxième occurrence à la fin d'une P2) à borner celle-ci vers la droite pour en améliorer la visibilité et faciliter d'autant le travail de décodage. 
De toutes façons, ce sont des opérations et des marquages très divers qui collaborent, chacun à son rang fonctionnel, pour instaurer cette relation subordonnante qui fait la P2 « de circonstance » : parmi lesquels ce morphème segmental dit « conjonction » n'est ni partout présent, ni structurellement nécessaire.

\section{BIBLIOGRAPHIE}

\section{Références des textes du corpus}

RAHAJARIZAFY A. (1960) Essai sur la Grammaire malgache, Antananarivo, Antanimena.

RAHAJARIZAFY A. Initiation à la langue malgache dans les lignes de l'enseignement du R.P. Antoine RAHAJARIZAFY, Antananarivo, Scolasticat Saint-Paul, Tsaramaso-andro.

RAJAONA S. éd. (1969) Takelaka notsongaina, II Lahatsoratra tsotra, Fianarantsoa, Ambozontany.

RAJEMISA-RAOLISON R. (1969) Grammaire malgache, Fianarantsoa, Ambozontany (1º́d. 1959).

RAMAROSON L. et GIAMBRoNE N. (1973) Teto anivon' ny riaka, Fianarantsoa, Ambozontany, (1ºéd. 1960)

RAPATSALAHY P. (1984) Hanambady any Iarivo, Tananarive, éd. Takariva, 5º́d.

RATSIFANDRIHAMANANA-ANDRIAMAMPANDRY C. (1982) Lavakombarika, Antananarivo, Librairie mixte.

\section{NOTES}

1. Abréviations utilisées ici $: \mathrm{N}=$ nom $; \mathrm{V}=$ verbe $;$ Adj. = adjectif $; \mathrm{SN}=$ syntagme nominal ; suj. $=$ sujet $;$ c.d. $=$ complément direct $;$ préd. $=$ prédicat $; \mathrm{SP}=$ syntagme prépositionnel $;$ c.c. $=$ complément circonstanciel, circonstant $; \mathrm{P} 1=$ la principale (dans une $\mathrm{P}$ complexe) $\mathrm{P} 2=\mathrm{la}$ subordonnée insérée sous P1.

Pour le corpus, la référence des énoncés utilisés se trouve à la fin de l'article.

Je remercie ici Madame Hanta RAKOTOMAVO, professeur à l'ENS d'Antananarivo, d'avoir bien voulu me prêter sa compétence de malgachophone pour bien interpréter les énoncés produits dans cet article. 


\section{AUTEUR}

\section{HUGUETTE FUGIER}

Université Marc Bloch - Strasbourg II 